\title{
Effects of Hyperuricemia on Beta-Cell Function, Renal Function, and Lipid Panels of Patients with Diabetic Kidney Disease: A Gender-Based Retrospective Study
}

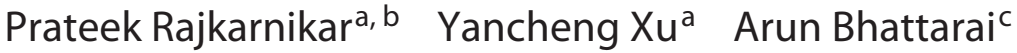 \\ aDepartment of Endocrinology, Wuhan University Zhongnan Hospital, Wuhan, China; ${ }^{b}$ Department of Internal \\ Medicine, Kirtipur Hospital, Kathmandu, Nepal; 'Department of Gastroenterology, Wuhan University Zhongnan \\ Hospital, Wuhan, China
}

\section{Keywords}

Serum uric acid - Normouricemia - Hyperuricemia - Diabetic kidney disease $\cdot$ Diabetic nephropathy

\begin{abstract}
Objective: Serum uric acid (SUA) has been linked with development and progression of diabetic kidney disease (DKD). In this study, we intend to compare the effects of hyperuricemia (HUA) on beta-cell function, renal function, and lipid panels of patients with DKD. Methods: A total of 492 patients with DKD were included for data collection and analysis. Males and females have different standard SUA levels; thus, we analyzed these 2 groups separately. Normouricemia (NUA) for men $(n=253)$ was $\leq 428 \mu \mathrm{mol} / \mathrm{L}$ and for women $(n=83)$ was $\leq 357 \mu \mathrm{mol} / \mathrm{L}$, whereas HUA for men $(n=94)$ was $>428 \mu \mathrm{mol} / \mathrm{L}$ and for women $(n=62)$ was $>357 \mu \mathrm{mol} / \mathrm{L}$. Clinical characteristics of patients were analyzed based on gender-specific NUA and HUA. The Spearman rank correlation test was used to evaluate the correlation between SUA and other clinical variables. Finally, stepwise multinomial logistic regression test was performed to identify the factors that are independently associated with HUA. Results: A total of 492 patients were included in this study. The regression analysis
\end{abstract}

karger@karger.com www.karger.com/dde

Karger"

BOPEN ACCESS
(C) 2020 The Author(s)

Published by S. Karger AG, Basel

This article is licensed under the Creative Commons AttributionNonCommercial-NoDerivatives 4.0 International License (CC BYNC-ND) (http://www.karger.com/Services/OpenAccessLicense). Usage and distribution for commercial purposes as well as any distribution of modified material requires written permission. showed that there was a significant association between HUA and decreased estimated glomerular filtration rate (eGFR) in both male and female patients (odds ratio (OR) [95\% confidence interval $(\mathrm{Cl})]=4.73[2.19-10.24], p$ value $\leq 0.01$ in male patients and $\mathrm{OR}[95 \% \mathrm{Cl}]=3.07[], p$ value $=$ 0.04 in female patients). FBG, $2 \mathrm{hPBG}$, and $\mathrm{HbA} 1 \mathrm{c}$ were negatively correlated with SUA in male patients $(r=-0.182, p$ value $\leq 0.01 ; r=-0.168, p$ value $\leq 0.01$; and $r=-0.187, p$ value $\leq 0.01$, respectively), whereas fasting insulin was positively correlated in male patients $(r=0.131, p$ value $=0.023)$ and female patients $(r=0.192, p$ value $=0.041)$. The atherogenic index of plasma was significantly high in patients with HUA (OR $[95 \% \mathrm{Cl}]=5.75[2.32-14.23], p$ value $\leq 0.01$ in male patients and $\mathrm{OR}[95 \% \mathrm{Cl}]=8.37[1.96-35.78], p$ value $\leq 0.01$ in female patients). Other indices of lipid profile such as lipoprotein combine index, atherosclerosis index, and triglyceride/high-density lipoprotein ratio were also independently associated with HUA in both male and female patients. Conclusion: SUA can affect various clinical parameters in patients with DKD. There is a significant association between HUA and decline in eGFR in both male and female patients. HUA is also associated with dyslipidemia in DKD, increasing the risk of cardiac complications and mortality.

(C) 2020 The Author(s)

Published by S. Karger AG, Basel

Prateek Rajkarnikar

Department of Internal Medicine, Kirtipur Hospital

Kirtipur, Nayabazaar

Kathmandu Bagmati 44618 (Nepal)

prateek627@gmail.com 


\section{Introduction}

Diabetic kidney disease (DKD), formerly known as diabetic nephropathy (DN), develops in approximately $40 \%$ of all diabetes (DM) patients and is recognized as the major microvascular complication of diabetes [1]. DN is defined as diabetes with albuminuria, impaired glomerular filtration rate (GFR), or both. Today, DKD includes not only DN but also atherosclerotic nephropathy, ischemic nephropathy, and interstitial fibrosis as consequences of diabetes [2]. The typical manifestations of $\mathrm{DN}$ include long-term duration of diabetes, retinopathy, proteinuria without gross hematuria, and progressive loss of estimated glomerular filtration rate (eGFR). However, in type 2 $\mathrm{DM}$, signs of CKD may appear in the absence of retinopathy, and a reduction in eGFR is often reported without albuminuria in both type 1 and type 2 DM [3]. With the escalating incidence of DM, DKD at present is the foremost cause of ESRD. In DKD, the kidney goes through quite a lot of changes, starting from deposition of extracellular matrix, glomerular basement membrane thickening (described by Mauer et al. [4] as the first histological sign of $\mathrm{DKD}$ ), proliferative alteration, tubular degeneration, to finally inducing interstitial fibrosis and glomerulosclerosis [5]. These abnormalities remain clinically silent for many years. As a result, with slow progression of DKD, until DKD is clinically portrayed by albuminuria and loss of renal function, renal parenchymal damage has already occurred. Therefore, it is essential to discover new DN risk biomarkers before albuminuria or renal dysfunction occurs for better early treatment. Many biomarkers associated with renal structural lesions with waning kidney function have been considered in recent years. One of them is serum uric acid (SUA). SUA independently amplifies the likelihood of new-onset kidney disease [6]. SUA has been linked with reduced GFR and augments the possibility of early progressive DKD in DM [7]. The impact of microinflammation on renal vascular function leads to endothelial damage provoked by SUA, suggesting that SUA affecting renal diseases may be conciliated by inflammatory response. Although ESRD may be the most obvious end result of DKD, most patients in reality die of cardiovascular events and infection before they receive renal replacement therapy. The increase in VLDL, low-density lipoproteins (LDL), and triglycerides (TG) and the decrease in cardioprotective high-density lipoproteins (HDL) are the core risk aspects of cardiovascular disease in T2DM. This abnormal lipid metabolism is mainly caused by insulin resistance and long-term hyperglycemia [8]. It is reported that elevated SUA is also linked with dyslipidemia, which is one of the main causes of cardiovascular complications in T2DM [9]. Different indices of lipid profile are used to determine the risk and severity of dyslipidemia other than classical LDL, HDL, TG, and total cholesterol (TC). One of the popular indices is the atherogenic index of plasma (AIP), which is a strong and independent predictor for atherosclerosis and coronary artery disease in the Chinese population [10]. Another index is the atherosclerosis index (AI), also known as non-HDL cholesterol (HDL-c)/HDL-c ratio; an AI more than 5 has a 6-fold risk of CAD [11]. Lipoprotein combine index (LCI) is also a marker of coronary heart diseases [11]. An independent relationship between SUA and islet dysfunction has never been confirmed. However, a cross-sectional study done at Zhongnan Hospital of Wuhan University demonstrated that SUA has a positive correlation with insulin secretion and insulin resistance indices in newly diagnosed T2DM patients [12], and insulin resistance is significantly associated with development of DN in type 2 diabetic patients. These associations of SUA with renal function, cardiovascular function, and beta-cell function of patients with DM have been well studied but not much with DKD in particular. In addition, standard SUA levels for men and women are different, so we conducted a gender-based retrospective study to compare the effects of hyperuricemia (HUA) on beta-cell function, renal function, and lipid panels of patients with DKD.

\section{Materials and Methods}

The study was designed as a retrospective study and included 492 patients (mean age $61.18 \pm 12.4$ years; male, $n=347$ [70.5\%], and female, $n=145$ [29.5\%]) diagnosed with DKD and admitted at the Department of Endocrinology of Zhongnan Hospital of Wuhan University from 2015 to 2018.

\section{Inclusion Criteria}

All patients diagnosed with DKD and admitted at the Department of Endocrinology, Zhongnan Hospital of Wuhan University from 2015 to 2018 were eligible for inclusion.

\section{Exclusion Criteria}

The exclusion criteria included the following:

Patients with type $1 \mathrm{DM}$, gestational diabetes, MODY, or LADA.

Patients who were pregnant.

Patients with biopsy-proven other existing kidney diseases or with the following features:

a an active urinary sediment (containing RBCs or white blood cells or cellular casts)

b rapidly increasing or nephrotic-range albuminuria $(>2,500$ $\mathrm{mg} / \mathrm{g}$ )

c rapidly decreasing eGFR 
Table 1. Comparison of demographic variables in male patients

\begin{tabular}{lccc}
\hline Variable & NUA $(n=253)$ & HUA $(n=94)$ & $p$ value \\
\hline Age, years & $59.7 \pm 11.8$ & $59.6 \pm 14.8$ & 0.942 \\
BMI, kg/m & $25.3 \pm 3.7$ & $25.7 \pm 4.1$ & 0.567 \\
DD, years & $8.3 \pm 6.9$ & $8.7 \pm 7.9$ & 0.654 \\
HTN, $n(\%)$ & $144(56.9)$ & $63(67.02)$ & 0.056
\end{tabular}

Data are expressed as mean $\pm \mathrm{SD}$, number (percentage), and median (range). DD, diabetes duration; HTN, hypertension; HUA, hyperuricemia; NUA, normouricemia.

Patients with ESRD.

Patients with renal stones and obstructive uropathy with prostate enlargement.

All patients with DM who had other reasons for elevated uric acid were excluded, which are given as follows:

a receiving cancer treatments

b patients with blood disorders

c patients taking medication for gout before admission

\section{Data Collection}

Data collection was done from records of Department of Endocrinology, Zhongnan Hospital of Wuhan University. The data collection technique used was through handwritten records, collecting variables on specific objectives. Demographic variables including age, gender, BMI, duration of DM, history of hypertension, and biochemical parameters including LDL, HDL, TC, TG, HbA1c, BUN, serum $\mathrm{Cr}$, urine albumin of timed (overnight) collection of urine, uric acid, fasting glucose, $2 \mathrm{~h}$ postprandial glucose, and fasting insulin were collected. Homeostasis model assessment of $\beta$-cell function (HOMA- $\beta$ ) and homeostasis model assessment, insulin resistance index (HOMA-IR) were calculated to evaluate $\beta$-cell function.

\section{Definition and Calculation}

Clinical diagnosis of DKD was made based on the presence of albuminuria and/or reduced eGFR in the absence of signs or symptoms of other primary causes of kidney damage. HOMA-IR was used to determine insulin sensitivity and HOMA- $\beta$ was used to determine insulin secretion. The eGFR was calculated by the abbreviated MDRD equation, and lipid profile indices such as AIP, AI, and LCI were also calculated. Formulas used for calculations are as follows:

HOMA-IR $=$ Fasting insulin $\times$ Fasting plasma glucose $/ 22.5$

HOMA $-\beta=(20 \times$ Fasting insulin $) /($ Fasting plasma glucose $-3.5)$

$\mathrm{eGFR}=186 \times(\mathrm{Cr} / 88.4)^{-1.154} \times(\text { Age })^{-0.203} \times(0.742$ if female $) \times$

(1.210 if black)

AIP $=\log (\mathrm{TG} / \mathrm{HDL})$

$\mathrm{AI}=(\mathrm{TC}-\mathrm{HDL}) / \mathrm{HDL}$

$\mathrm{LCI}=\mathrm{TC} \times \mathrm{TG} \times \mathrm{LDL} / \mathrm{HDL}$

\section{Statistical Analysis}

The Statistical Package for Social Science software (SPSS) version 19.0 was used to perform all statistical analyses. Baseline clinical characteristics were assessed with standard descriptive statis-
Table 2. Comparison of demographic variables in female patients

\begin{tabular}{lccc}
\hline Variable & NUA $(n=83)$ & HUA $(n=62)$ & $p$ value \\
\hline Age, years & $63.2 \pm 10.9$ & $66.48 \pm 11$ & 0.08 \\
BMI, kg/m & $25.4 \pm 4.3$ & $25.7 \pm 4.2$ & 0.789 \\
DD, years & $9.0 \pm 6.8$ & $9.9 \pm 7.2$ & 0.476 \\
HTN, $n(\%)$ & $46(55.4)$ & $48(77.4)$ & $<0.01$
\end{tabular}

Data are expressed as mean $\pm \mathrm{SD}$, number (percentage), and median (range). DD, diabetes duration; HTN, hypertension; HUA, hyperuricemia; NUA, normouricemia. tics. All continuous variables were presented as mean \pm SD or as median (ranges), and categorical variables were presented as number or percentage. As men and women have different average SUA levels, we analyzed these 2 groups separately. Normouricemia (NUA) for men $(n=253)$ was considered as $\leq 428 \mu \mathrm{mol} / \mathrm{L}$ and for women $(n=83)$ as $\leq 357 \mu \mathrm{mol} / \mathrm{L}$, whereas HUA for men $(n=94)$ was considered as $>428 \mu \mathrm{mol} / \mathrm{L}$ and for women $(n=62)$ as $>357$ $\mu \mathrm{mol} / \mathrm{L} . \chi^{2}$ tests were performed for comparing categorical variables between groups, and independent sample $t$ test was used for among-group comparisons. If data were not normally distributed or did not meet the homogeneity of variances, the Mann-Whitney test was used. Pearson and Spearman rank correlation tests were used to assess the correlation between SUA and other variables. Finally, stepwise binary logistic test was carried out to evaluate the factors that are independently associated with an increase in SUA. Odds ratio (OR) with confidence interval (CI) of $95 \%$ after adjusting for potential confounding factors were used to express results. A 2 -tailed $p$ value $<0.05$ was considered statistically significant.

\section{Results}

\section{Demographic and Clinical Characteristics of Study Patients}

There were a total of 492 patients (mean age $61.18 \pm$ 12.4 years; male, $n=347$ [70.5\%] and female, $n=145$ [29.5\%]) enrolled for this study. Clinical characteristics of male patients according to NUA and HUA are shown in Table 1, and clinical characteristics of female patients according to NUA and HUA are shown in Table 2. Comparison of demographic variables in male and female DKD patients did not show any significant difference except for history of hypertension in female patients ( $p$ value $<0.01)$. Comparison of $\beta$-cell function in male and female patients is shown in Tables 3 and 4, respectively. FBG, 2hPBG, and HbA1c were significantly low in male patients with HUA, while no significant difference was seen in female patients. Comparisons of renal function in male and female patients are shown in Tables 5 and 6, respectively. BUN and Scr were significantly high in both 
Table 3. Comparison of $\beta$-cell function in male patients

\begin{tabular}{lccc}
\hline Variable & NUA $(n=253)$ & HUA $(n=94)$ & $p$ value \\
\hline FBG, mmol/L & $10.1 \pm 3.9$ & $8.8 \pm 4.3$ & 0.010 \\
2hPBG, mmol/L & $18.8 \pm 5.8$ & $17.3 \pm 6.0$ & 0.040 \\
Fasting insulin, IU/mL & $8.5(0.1,300)$ & $10.7(1.3,137.0)$ & 0.682 \\
HbAlc, \% & $8.9 \pm 2.2$ & $8.2 \pm 2.1$ & $<0.01$ \\
HOMA-IR & $3.6(0.1,149.8)$ & $3.8(0.2,118.2)$ & 0.697 \\
HOMA- $\beta$ & $28.0(-464.2,2,105.2)$ & $48.7(-38.0,740.5)$ & 0.246 \\
\hline
\end{tabular}

Data are expressed as mean $\pm \mathrm{SD}$, number (percentage), and median (range). FBG, fasting blood glucose; PBG, postprandial blood glucose; HOMA-IR, homeostasis model assessment insulin resistance; HOMA- $\beta$, homeostasis model assessment, insulin secretion; HUA, hyperuricemia; NUA, normouricemia.

Table 4. Comparison of $\beta$-cell function in female patients

\begin{tabular}{lccc}
\hline Variable & NUA $(n=83)$ & HUA $(n=62)$ & $p$ value \\
\hline FBG, mmol/L & $9.8 \pm 3.9$ & $10.8 \pm 4.7$ & 0.173 \\
2hPBG, mmol/L & $19.2 \pm 5.7$ & $19.2 \pm 6.4$ & 0.967 \\
Fasting insulin, IU/mL & $12.9 \pm 10.5$ & $14.2 \pm 9.1$ & 0.513 \\
HbAlc, $\%$ & $8.8 \pm 2.0$ & $8.9 \pm 1.7$ & 0.726 \\
HOMA-IR & $4.2(02,38.0)$ & $4.9(0.6,28.1)$ & 0.458 \\
HOMA- $\beta$ & $38.3(3.2,349.1)$ & $30.9(-1,600.0,2,662.8)$ & 0.812 \\
\hline
\end{tabular}

Data are expressed as mean \pm SD, number (percentage), and median (range). FBG, fasting blood glucose; PBG, postprandial blood glucose; HOMA-IR, homeostasis model assessment, insulin resistance; HOMA- $\beta$, homeostasis model assessment, insulin secretion; HUA, hyperuricemia; NUA, normouricemia.

Table 5. Comparison of renal function in male patients

\begin{tabular}{lccc}
\hline Variable & $\begin{array}{l}\text { NUA } \\
(n=253)\end{array}$ & $\begin{array}{l}\text { HUA } \\
(n=94)\end{array}$ & $p$ value \\
\hline Urine albumin, $\mu \mathrm{g} / \mathrm{min}$ & $50.8 \pm 25.6$ & $54.2 \pm 26.4$ & 0.327 \\
BUN, $\mathrm{mmol} / \mathrm{L}$ & $6.1 \pm 2.2$ & $7.3 \pm 3.2$ & $<0.01$ \\
Scr, $\mu \mathrm{mol} / \mathrm{L}$ & $80.5 \pm 32.6$ & $108.0 \pm 54.9$ & $<0.01$ \\
eGFR, $\mathrm{mL} / \mathrm{min}$ per $1.73 \mathrm{~m}^{2}$ & $105.3 \pm 38.4$ & $82.0 \pm 39.8$ & $<0.01$ \\
\hline
\end{tabular}

Data are expressed as mean $\pm \mathrm{SD}$, number (percentage), and median (range). Scr, serum Cr; eGFR, estimated glomerular filtration rate; HUA, hyperuricemia; NUA, normouricemia.

male and female patients, whereas eGFR was significantly decreased with an increase in uric acid levels. Comparisons of lipid profile in male and female patients are shown in Tables 7 and 8, respectively. AI, AIP, LCI, TG/ HDL ratio, and TG were significantly high in both male and female patients with HUA. While HDL was low in female patients with HUA, no significant difference was
Table 6. Comparison of renal function in female patients

\begin{tabular}{lccc}
\hline Variable & $\begin{array}{l}\text { NUA } \\
(n=83)\end{array}$ & $\begin{array}{l}\text { HUA } \\
(n=62)\end{array}$ & $p$ value \\
\hline Urine albumin, $\mu \mathrm{g} / \mathrm{min}$ & $50.2 \pm 29.6$ & $65.3 \pm 27.7$ & 0.005 \\
BUN, $\mathrm{mmol} / \mathrm{L}$ & $5.5 \pm 2.0$ & $7.8 \pm 2.9$ & $<0.01$ \\
Scr, $\mu \mathrm{mol} / \mathrm{L}$ & $63.6 \pm 32.2$ & $94.7 \pm 60.7$ & $<0.01$ \\
eGFR, $\mathrm{mL} / \mathrm{min}$ per $1.73 \mathrm{~m}^{2}$ & $105.1 \pm 38.8$ & $79.0 \pm 46.8$ & $<0.01$ \\
\hline
\end{tabular}

Data are expressed as mean $\pm \mathrm{SD}$, number (percentage), and median (range). Scr, serum Cr; eGFR, estimated glomerular filtration rate; HUA, hyperuricemia; NUA, normouricemia.

seen in the LDL level between normal and HUA in both male and female patients.

\section{Correlations between SUA and Various Parameters by} Gender

Pearson and Spearman rank correlation tests were used to assess the correlation between SUA and other 
Table 7. Comparison of lipid profile in male patients

\begin{tabular}{lllc}
\hline Variable & NUA $(n=253)$ & HUA $(n=94)$ & $p$ value \\
\hline HDL-c, mmol/L & $1.0 \pm 0.2$ & $1.0 \pm 0.3$ & 0.784 \\
LDL-c, mmol/L & $2.7 \pm 0.9$ & $2.7 \pm 0.9$ & 0.805 \\
TG, mmol/L & $2.2 \pm 1.8$ & $3.1 \pm 2.8$ & $<0.01$ \\
TC, mmol/L & $4.4 \pm 1.1$ & $4.9 \pm 1.3$ & $<0.01$ \\
TG/HDL ratio & $1.6(0.2,19.7)$ & $2.6(0.3,30.3)$ & $<0.01$ \\
AI & $3.4 \pm 1.3$ & $4.1 \pm 2.1$ & $<0.01$ \\
AIP & $0.2(-0.5,1.2)$ & $0.4(-0.4,1.4)$ & $<0.01$ \\
LCI & $30.8(0.7,189.3)$ & $30.6(2.3,668.0)$ & $<0.01$ \\
\hline
\end{tabular}

Data are expressed as mean $\pm \mathrm{SD}$, number (percentage), and median (range). TC, total cholesterol; TG, triglycerides; HDL-c, high-density lipoprotein-cholesterol; LDL, low-density lipoprotein-cholesterol; AIP, atherogenic index of plasma; AI, atherosclerosis index; LCI, lipoprotein combine index; HUA, hyperuricemia; NUA, normouricemia.
Table 8. Comparison of lipid profile in female patients

\begin{tabular}{llcc}
\hline Variable & NUA $(n=83)$ & HUA $(n=62)$ & $p$ value \\
\hline HDL-c, mmol/L & $1.1 \pm 0.3$ & $1.0 \pm 0.2$ & 0.043 \\
LDL-c, mmol/L & $2.8 \pm 0.9$ & $2.6 \pm 0.9$ & 0.239 \\
TG, mmol/L & $2.3 \pm 1.8$ & $3.4 \pm 2.8$ & 0.007 \\
TC, mmol/L & $4.8 \pm 1.1$ & $5.0 \pm 1.5$ & 0.288 \\
TG/HDL ratio & $2.3 \pm 2.5$ & $3.7 \pm 3.4$ & $<0.01$ \\
AI & $3.3 \pm 1.3$ & $4.0 \pm 1.8$ & 0.011 \\
AIP & $0.1(-0.7,1.1)$ & $0.3(-0.3,1.1)$ & $<0.01$ \\
LCI & $21.5(0.9,199.7)$ & $35.3(3.4,337.3)$ & 0.020 \\
\hline
\end{tabular}

Data are expressed as mean $\pm \mathrm{SD}$, number (percentage), and median (range). TC, total cholesterol; TG, triglycerides; HDL-c, high-density lipoprotein-cholesterol; LDL, low-density lipoprotein-cholesterol; AIP, atherogenic index of plasma; AI, atherosclerosis index; LCI, lipoprotein combine index; HUA, hyperuricemia; NUA, normouricemia.

Table 9. Correlation coefficients between SUA and various parameters

\begin{tabular}{|c|c|c|c|c|c|c|}
\hline & \multicolumn{2}{|c|}{ Overall $(n=492)$} & \multicolumn{2}{|c|}{ Male $(n=347)$} & \multicolumn{2}{|c|}{ Female $(n=145)$} \\
\hline & $r$ value & $p$ value & $r$ value & $p$ value & $r$ value & $p$ value \\
\hline Age, years & 0.549 & 0.194 & 0.051 & 0.345 & 0.111 & 0.185 \\
\hline $\mathrm{BMI}, \mathrm{kg} / \mathrm{m}^{2}$ & 0.129 & 0.044 & 0.122 & 0.110 & 0.148 & 0.218 \\
\hline DD, years & 0.028 & 0.531 & 0.030 & 0.578 & 0.030 & 0.719 \\
\hline $\mathrm{FBG}, \mathrm{mmol} / \mathrm{L}$ & -0.100 & 0.028 & -0.182 & $<0.01$ & 0.104 & 0.225 \\
\hline 2hPBG, mmol/L & -0.133 & $<0.01$ & -0.168 & $<0.01$ & -0.017 & 0.840 \\
\hline Fasting insulin, IU/mL & 0.134 & $<0.01$ & 0.131 & 0.023 & 0.192 & 0.041 \\
\hline $\mathrm{HbAlc}, \%$ & -0.134 & $<0.01$ & -0.187 & $<0.01$ & 0.020 & 0.817 \\
\hline Urine albumin, $\mu \mathrm{g} / \mathrm{min}$ & 0.186 & $<0.01$ & 0.122 & 0.040 & 0.315 & $<0.01$ \\
\hline $\mathrm{BUN}, \mathrm{mmol} / \mathrm{L}$ & 0.326 & $<0.01$ & 0.243 & $<0.01$ & 0.491 & $<0.01$ \\
\hline $\mathrm{Scr}, \mu \mathrm{mol} / \mathrm{L}$ & 0.423 & $<0.01$ & 0.383 & $<0.01$ & 0.476 & $<0.01$ \\
\hline eGFR, $\mathrm{mL} / \mathrm{min}$ per $1.73 \mathrm{~m}^{2}$ & -0.395 & $<0.01$ & -0.369 & $<0.01$ & -0.463 & $<0.01$ \\
\hline HOMA-IR & 0.100 & 0.042 & 1.53 & 0.023 & 0.200 & 0.034 \\
\hline HOMA- $\beta$ & 0.135 & $<0.01$ & 0.184 & $<0.01$ & 0.017 & 0.858 \\
\hline \multicolumn{7}{|l|}{ Lipid profile } \\
\hline $\mathrm{HDL}-\mathrm{c}, \mathrm{mmol} / \mathrm{L}$ & -0.178 & $<0.01$ & -0.163 & $<0.01$ & -0.183 & 0.031 \\
\hline LDL-c, mmol/L & -0.68 & 0.139 & -0.18 & 0.736 & -0.180 & 0.035 \\
\hline $\mathrm{TG}, \mathrm{mmol} / \mathrm{L}$ & 0.251 & $<0.01$ & 0.232 & $<0.01$ & 0.308 & 0.070 \\
\hline $\mathrm{TC}, \mathrm{mmol} / \mathrm{L}$ & 0.087 & 0.058 & 0.114 & 0.036 & 0.303 & $<0.01$ \\
\hline TG/HDL ratio & 0.261 & $<0.01$ & 0.239 & $<0.01$ & 0.313 & $<0.01$ \\
\hline AI & 0.197 & $<0.01$ & 0.206 & $<0.01$ & 0.173 & 0.043 \\
\hline AIP & 0.261 & $<0.01$ & 0.239 & $<0.01$ & 0.303 & $<0.01$ \\
\hline LCI & 0.211 & $<0.01$ & 0.214 & $<0.01$ & 0.224 & $<0.01$ \\
\hline
\end{tabular}

SUA, serum uric acid; AI, atherosclerosis index; AIP, atherogenic index of plasma; LCI, lipoprotein combine index; eGFR, estimated glomerular filtration rate; HOMA-IR, homeostasis model assessment, insulin resistance; HOMA- $\beta$, homeostasis model assessment, insulin secretion; HDL, high-density lipoproteins; LDL, low-density lipoproteins. 
Table 10. Logistic regression analysis of factors associated with SUA in male patients

\begin{tabular}{|c|c|c|c|c|}
\hline \multirow[t]{2}{*}{ Variable } & \multirow[t]{2}{*}{$\mathrm{NUA} \leq 428 \mu \mathrm{mol} / \mathrm{L}$} & \multicolumn{3}{|c|}{$\mathrm{HUA}>428 \mu \mathrm{mol} / \mathrm{L}$} \\
\hline & & OR & $95 \% \mathrm{CI}$ & $p$ value \\
\hline Decreased eGFR & Reference & 4.73 & $2.19-10.24$ & $<0.01$ \\
\hline TG & Reference & 1.29 & $1.13-1.48$ & $<0.01$ \\
\hline TC & Reference & 1.51 & $1.19-1.91$ & $<0.01$ \\
\hline AIP & Reference & 5.75 & $2.32-14.23$ & $<0.01$ \\
\hline AI & Reference & 1.38 & $1.13-1.68$ & $<0.01$ \\
\hline LCI & Reference & 1.01 & $1.00-1.02$ & $<0.01^{\star}$ \\
\hline TG/HDL ratio & Reference & 1.17 & $1.06-1.29$ & $<0.01$ \\
\hline
\end{tabular}

SUA, serum uric acid; HUA, hyperuricemia; NUA, normouricemia; AI, atherosclerosis index; AIP, atherogenic index of plasma; LCI, lipoprotein combine index; eGFR, estimated glomerular filtration rate; HDL, high-density lipoproteins; OR, odds ratio; $\mathrm{CI}$, confidence interval. ${ }^{*}$ Note that the null value of the confidence interval for the OR is one. If the $95 \%$ CI for the OR includes the null value of 1 , then there is insufficient evidence to conclude that the groups are statistically significantly different.

Table 11. Logistic regression analysis of factors associated with SUA in female patients

\begin{tabular}{|c|c|c|c|c|}
\hline \multirow[t]{2}{*}{ Variable } & \multirow[t]{2}{*}{$\mathrm{NUA} \leq 357 \mu \mathrm{mol} / \mathrm{L}$} & \multicolumn{3}{|c|}{$\mathrm{HUA}>357 \mu \mathrm{mol} / \mathrm{L}$} \\
\hline & & OR & $95 \%$ CI & $p$ value \\
\hline Decreased eGFR & Reference & 3.07 & $1.01-9.32$ & 0.04 \\
\hline TG & Reference & 1.35 & $1.05-1.73$ & 0.017 \\
\hline HDL & Reference & 0.15 & $0.03-0.70$ & 0.016 \\
\hline AIP & Reference & 8.37 & $1.96-35.78$ & $<0.01$ \\
\hline $\mathrm{AI}$ & Reference & 1.55 & $1.15-2.09$ & $<0.01$ \\
\hline LCI & Reference & 1.01 & $1.00-1.02$ & $0.013^{\star}$ \\
\hline TG/HDL ratio & Reference & 1.22 & $1.02-1.46$ & 0.02 \\
\hline
\end{tabular}

SUA, serum uric acid; HUA, hyperuricemia; NUA, normouricemia; AI, atherosclerosis index; AIP, atherogenic index of plasma; LCI, lipoprotein combine index; eGFR, estimated glomerular filtration rate; OR, odds ratio; CI, confidence interval; HDL, high-density lipoproteins. ${ }^{*}$ Note that the null value of the CI for the OR is 1 . If the $95 \%$ CI for the OR includes the null value of 1 , then there is insufficient evidence to conclude that the groups are statistically significantly different.

variables. The tests were performed 3 times in relation to the overall study patients, male patients, and female patients separately, as shown in Table 9. FBG, 2hPBG, and $\mathrm{HbA} 1 \mathrm{c}$ were negatively correlated with SUA in male patients $(r=-0.182, p$ value $\leq 0.01 ; r=-0.168$, $p$ value $\leq 0.01$; and $r=-0.187$, $p$ value $\leq 0.01$, respectively), whereas fasting insulin was positively correlated in male patients $(r=$ $0.131, p$ value $=0.023)$ and female patients $(r=0.192, p$ value $=0.041)$. HOMA-IR was positively associated with SUA in both male $(r=1.53$, $p$ value $=0.02)$ and female DKD patients $(r=0.2, p$ value $=0.03)$. HOMA- $\beta$ was positively associated with SUA in male patients $(r=$ $0.184, p$ value $\leq 0.01)$. Urine albumin was positively as- sociated with SUA in both male patients $(r=0.122, p$ value $=0.04)$ and in female patients $(r=0.315, p$ value $\leq 0.01)$. Scr and BUN were positively associated with an increase in SUA in both male and female patients. eGFR was significantly low in HUA in both male $(r=-0.369, p$ value $\leq 0.01)$ and female patients $(r=-0.463, p$ value $\leq 0.01)$. HDL was negatively associated with SUA in both male $(r=-0.163, p$ value $\leq 0.01)$ and female patients $(r=$ -0.183 , $p$ value $=0.03)$, whereas LDL was significantly associated with SUA only in female patients $(r=-0.183$, $p$ value $=0.03)$. TG, TC, TG/HDL ratio, AI, AIP, and LCI were all positively associated with SUA in both male and female patients. 


\section{Logistic Regression Analysis}

Multinomial logistic regression test was carried out to evaluate the factors that are independently associated with an increase in SUA. OR with CI of 95\% after adjusting for potential confounding factors was used to express results (Tables 10 and 11). Independent variables such as age, TC, TG, HDL-c, LDL-c, eGFR, history of HTN, FBG, $2 \mathrm{hPBG}, \mathrm{HbA1c}$, and urine albumin were enrolled as potential confounding factors. HUA was significantly associated with a decrease in eGFR in both male (OR [95\% $\mathrm{CI}]=4.73[2.19-10.24], p$ value $\leq 0.01)$ and female DKD patients $(\mathrm{OR}[95 \% \mathrm{CI}]=3.07[1.01-9.32], p$ value $=0.04)$. Lipid profile indices such as AIP, AI, LCI, and TG/HDL ratio are all independently associated with high SUA. However, since the CI of LCI in both male and female patients includes the null value of 1 , there is insufficient evidence to conclude that the value of LCI is statistically significantly different in high and normal SUA.

\section{Discussion}

$\mathrm{DKD}$ is the principal cause of ESRD requiring dialysis and kidney transplant in severe cases. In addition, DKD has been extensively linked with cardiovascular complications and mortality. Studies have been focusing on early diagnosis of $\mathrm{DKD}$ so as to avert the progression to ESRD. Pearson and Spearman rank correlation tests showed eGFR was inversely correlated with SUA ( $r=$ $-0.395, p$ value $\leq 0.01)$. There are different normal uric acid ranges for men and women. SUA levels in adult women are lower than those in men of the same age and are thought to be associated with higher uric acid clearance rates in women, possibly because of their higher plasma estrogen levels [13]. In this study, we found that SUA was significantly associated with decreased eGFR in both men and women. The regression analysis showed that HUA was significantly associated with decrease in eGFR in male (OR [95\% CI] $=4.73$ [2.19-10.24], $p$ value $\leq 0.01$ ) and female patients (OR $[95 \% \mathrm{CI}]=3.07[1.01-$ 9.32], $p$ value $=0.04$ ). Uric acid is mainly eliminated by the kidney, and the decrease in eGFR is almost generally related to the rise in SUA [14]. On the contrary, uric acid can provoke the commencement of RAAS and endothelial dysfunction, amplify oxidative stress, and cause inflammation, leading to the decrease in eGFR and tubulointerstitial fibrosis. In T2DM, HUA seems to be associated with insulin resistance syndrome and an increase in early onset or progression to nephropathy [15]. Thus, an elevated uric acid is strongly related to the development

Effects of Hyperuricemia in Patients with DKD of DKD. We excluded patients with grade 4 kidney failure and ESRD patients to avoid confounding effects.

We found that the risk of dyslipidemia is higher in patients with higher SUA in DKD patients. Contrary to previous studies, we could not demonstrate the classic high TG, high LDL, and low HDL in both male and female patients. However, when we used different indices of lipid profiles such as AIP, AI, LCI, and TG/HDL ratio, there were significant results in both male and female patients. AIP was significantly high in in male (OR $[95 \% \mathrm{CI}]=5.75$ [2.32-14.23], $p$ value $\leq 0.01)$ and female patients with HUA $($ OR $[95 \% \mathrm{CI}]=8.37$ [1.96-35.78], $p$ value $\leq 0.01)$. $\mathrm{AI}$, also known as non-HDL-c/HDL-c ratio, has a 6 -fold risk of CAD if the value is more than 5 [11]; it was also high in both male and female patients with HUA (OR $[95 \% \mathrm{CI}]=1.38[1.13-1.68], p$ value $\leq 0.01$ in males and OR $[95 \% \mathrm{CI}]=1.55$ [1.15-2.09], $p$ value $\leq 0.01$ in females). LCI, which is also a marker of coronary heart diseases [11], was also elevated in male and female patients with HUA $($ OR $[95 \% \mathrm{CI}]=1.01[1.00-1.02], p$ value $\leq 0.01$ in males and OR $[95 \% \mathrm{CI}]=1.01[1.00-1.02], p$ value $=0.013$ in females). However, since the CI of LCI in both male and female patients includes the null value of 1 , there is insufficient evidence to conclude that the value of LCI is statistically significantly different in high and normal SUA. More studies are needed to confirm the association of LCI and SUA in DKD patients.

Finally, a similar increase in TG/HDL ratio, which is a reliable indicator of insulin resistance, was seen in male and female patients (OR [95\% CI] $=1.17$ [1.06-1.29], $p$ value $\leq 0.01$ in males and $\mathrm{OR}[95 \% \mathrm{CI}]=1.22$ [1.02-1.46], $p$ value $=0.02$ in females). Studies have shown the relation between SUA and lipid levels [16]. In this study, we demonstrated that patients with DKD are at more risk of cardiovascular complications when accompanied by high SUA levels. We also found that individual levels of TC, TG, LDL, and HDL might be misguiding, and the use of various indices of lipid profile would provide more significant information.

Overall, correlation coefficient tests showed that DKD patients with higher BMI tend to have a higher level of SUA $(r=0.129, p=0.04)$. In China, obesity is common in $\mathrm{DKD}$ patients. Obesity contributes in greater proteinuria at an early stage, while weight loss is seen in the later stage of ESRD [17]. Urine albumin excretion (UAE) is the most widely and commonly used marker for screening and diagnosis of DKD. Albumin excretion is an indicator of kidney damage in DKD. In our study, we found that urine albumin increases with an increase in SUA levels $(r=$ 0.186 , $p$ value $\leq 0.01$ ).

Dubai Diabetes Endocrinol J 2020;26:103-11 109 
Fasting insulin secretion increased with rising SUA levels in both men and women. Correlation coefficient tests showed the positive correlation between fasting insulin and uric acid in both male $(r=0.131, p$ value $=$ $0.023)$ and female patients $(r=0.192, p$ value $=0.041)$. The mechanism of SUA in insulin resistance and insulin secretion is unclear in many animal and clinical studies. Prior studies have revealed that HUA and insulin resistance support each other. In particular, hyperinsulinemia facilitates the reabsorption of uric acid and sodium ions in the brush border membranes of proximal renal tubules [18].Our study supports this fact that many studies have shown a positive relation of increased insulin secretion with increase in SUA [19]. Previous studies have also failed to provide a solid evidence of association between SUA and blood glucose levels. Overall, previous studies have explained the negative correlation of $\mathrm{HbA1c}$, stating that the correlation between uric acid and $\mathrm{HbA} 1 \mathrm{c}$ most likely relies on insulin levels. This may be due to the effect of insulin on uric acid and glucose metabolism. Hyperinsulinemia can increase the activation of hexose phosphate shunt, promote the biosynthesis and transformation of purine, and thus improve the urinary uric acid excretion rate [20]. In another study done on 7,735 middle-aged men, the serum glucose level was positively correlated with SUA concentration, up to $8 \mathrm{mmol} / \mathrm{L}$. At higher levels of glucose, SUA decreased. Uric acid levels were significantly reduced in insulindependent diabetics, in those on oral hypoglycemics, and also in "non-diabetics" with casual glucose levels $>10 \mathrm{mmol} / \mathrm{L}$. This reflects the biochemical interaction between blood sugar and purine metabolism, and uric acid excretion increased during hyperglycemia and glycuria [21]. Our study agrees with these facts as there was a negative correlation between FBG, 2hPBG, and HbAlc in our study.

Currently, almost all guidelines have recommended diagnosing and treating DKD on the basis of UAE levels and evaluating the progression depending upon GFR. While it is clinically a more practical approach, many researchers debate that DKD can be diagnosed before the appearance of microalbumin. While many biomarkers have been studied and claimed to be a novel marker of early diagnosis of $\mathrm{DKD}$, many of them are not yet clinically applicable. However, SUA has shown a promising result as a novel independent biomarker of DKD. Considering the results of our study that HUA has a 4-fold risk of decreasing eGFR in male patients and a 3 -fold risk of decreasing eGFR in female patients with DKD, it warrants a need of board therapeutic management of DKD.
Also, the HUA can lead to severe dyslipidemia in patients with DKD, increasing the risk of cardiovascular complications and mortality. For SUA and eGFR, the question "which is chasing which?" is a debatable issue; however, we have enough evidence that SUA is involved in various stages of DKD onset and progression to nephropathy and finally ESRD [22]. There is evidence that lowering uric acid levels may play a role in part by blocking RAS. Uric acid stimulates angiotensin II production in vascular cells and increases renin expression in experimental animals [23]. A randomized open parallel-controlled study was performed in Qingdao University Affiliated Hospital, China, with 176 patients with T2DM and asymptomatic HUA. The result showed that after 3 years of treatment, allopurinol treatment could effectively reduce SUA, UAER, and $\mathrm{Cr}(p<0.01)$ and increase GFR $(p$ $<0.01$ ) compared with conventional treatment [24]. "The PERL Study: Preventing Early Renal Function Loss in Type 1 Diabetes" [25] was a double-blind, placebocontrolled, parallel group, randomized clinical trial conducted at 16 centers in the USA, Canada, and Denmark, which was expected to end in July 2019. Atorvastatin, a lipid-lowering drug, can also significantly lower SUA levels. This result shows beneficial findings to the treatment of hyperlipidemia patients with HUA by atorvastatin [26].

\section{Conclusion}

SUA can affect various clinical parameters in patients with DKD. Most significantly, there is a significant decrease in eGFR in both male and female patients with HUA. Dyslipidemia is also associated with HUA in DKD, increasing the risk of cardiac complications and mortality. SUA should be used alongside UAE as a novel biomarker to assess the progression and severity of renal function deterioration in DKD patients. However, further large-scale prospective studies are needed to evaluate the effect of SUA in progression of DKD and to study applicable treatment options.

\section{Acknowledgements}

The author would like to thank Professor Xu Yancheng and all the staff of the Department of Endocrinology of Wuhan University Zhongnan Hospital for helping and supporting throughout the residency program and conducting this research. 


\section{Statement of Ethics}

This research is a part of thesis research entitled "A Comparative study of Hyperuricaemia and Normouricaemia in patients with Type 2 Diabetic Kidney Disease" submitted by Prateek Rajkarnikar, at Wuhan University School of Medicine, Wuhan, China, in 2019 as partial fulfillment of the requirement for a master's degree in Clinical Internal Medicine and Endocrinology in accordance with the rules and regulations set by the School of Medicine of Wuhan University. The author declares that the work submitted for the thesis has been composed solely by himself and that it has not been submitted or published, in whole or in piece, elsewhere. The thesis work has been approved for publication by Wuhan University School of Medicine. All information were collected retrospectively from digital records. All patients were given serial numbers, and no names were recorded. The study protocol was approved by the Department of Endocrinology of Wuhan University Zhongnan Hospital.

\section{Conflict of Interest Statement}

The authors have no conflicts of interest to declare.

\section{Funding Sources}

We certify that this article has not been funded by any funding agency.

\section{Author Contributions}

All authors contributed to this study and the preparation of the manuscript.

\section{References}

1 Alicic RZ, Rooney MT, Tuttle KR. Diabetic kidney disease: challenges, progress, and possibilities. Clin J Am Soc Nephrol. 2017;12(12): 2032-45. Available from: https://cjasn.asnjournals.org/content/12/12/2032.http: //dx. doi.org/10.2215/CJN.11491116.

2 Toth-Manikowski S, Atta MG. Diabetic kidney disease: pathophysiology and therapeutic targets. J Diabetes Res. 2015;2015:697010.

3 Riddle MC. Standards of medical care in diabetes 2019 ADA. Am Diabetes Assoc 2019; 42(479):960-1010. https://care.diabetesjournals.org/content/diacare/suppl/2019/ 12/20/43.Supplement_1.DC1/Standards_of_ Care_2020.pdf.

4 Mauer SM, Steffes MW, Brown DM. Effects of mesangial localization of polyvinyl alcohols on glomerular basement membrane thickness. Kidney Int. 1985;27(5):751-5. Available from: http://dx.doi.org/10.1038/ki.1985.75.

5 Gnudi L, Coward RJM, Long DA. Diabetic nephropathy: perspective on novel molecular mechanisms. Trends Endocrinol Metab. 2016;27(11):820-30. Available from: http:// dx.doi.org/10.1016/j.tem.2016.07.002.

6 Obermayr RP, Temml C, Gutjahr G, Knechtelsdorfer M, Oberbauer R, Klauser-Braun R. Elevated uric acid increases the risk for kidney disease. J Am Soc Nephrol. 2008;19(12): 2407-13. Available from: http://www.jasn. org/cgi/doi/10.1681/ASN.2008010080.http:// dx.doi.org/10.1681/ASN.2008010080.

7 Pilemann-Lyberg S, Lindhardt M, Persson F, Andersen S, Rossing P. Serum uric acid and progression of diabetic nephropathy in type 1 diabetes. J Diabetes Complicat. 2018 May; 32(5):470-3.

8 Vergès B. Pathophysiology of diabetic dyslipidaemia: where are we? Diabetologia. 2015; 58(5):886-99.

9 Dias L, Malheiro J, Fonseca I, Almeida M, Pedroso S, Martins LS, et al. Hyperuricemia in adult renal allograft recipients: prevalence and predictors. Transplant Proc. 2012;44(8): 2369-72.

10 Cai G, Shi G, Xue S, Lu W. The atherogenic index of plasma is a strong and independent predictor for coronary artery disease in the Chinese Han population. Medicine. 2017; 96(37):e8058-6.

11 Li-hua HU, Zhi-tao JIN, Li-juan Z, Wei Y, Lina Z, Tao-hong HU, et al. Correlation between serum lipid parameters and coronary lesion severity. Chin J Evid Based Cardiovasc Med. 2015;7(3):323-5.

12 Hu Y, Liu J, Li H, Zhu H, Liu L. The association between elevated serum uric acid levels and islet b-cell function indexes in newly diagnosed type 2 diabetes mellitus: a cross-sectional study. PeerJ. 2018:1-17.

13 Mateos Antón F, García Puig J, Ramos T, González P, Ordás J. Sex differences in uric acid metabolism in adults: evidence for a lack of influence of estradiol-17 $\beta$ (E2) on the renal handling of urate. Metabolism 1986;35(4): 343-8.

14 Marangella M. Uric acid elimination in the urine. Pathophysiological implications. Contrib Nephrol. 2005;147:132-48.

15 Bo S, Cavallo-Perin P, Gentile L, Repetti E, Pagano G. Hypouricemia and hyperuricemia in type 2 diabetes: two different phenotypes. Eur J Clin Invest. 2001 Apr;31(4):318-21.

16 Biyik Z, Guney I. Relationship between uric acid, proteinuria, and atherogenic index of plasma in renal transplant patients. Transplant Proc. 2018 Dec;50(10):3376-80. Available from: http://www.sciencedirect.com/science/article/pii/S0041134518307267.

17 Chen HM, Shen WW, Ge YC, Zhang YD, Xie HL, Liu ZH. The relationship between obesity and diabetic nephropathy in China. BMC Nephrol. 2013 Mar 25;14:69. Available from: ht tps://www.ncbi.nlm.nih.gov/ pubmed/23521842.http://dx.doi.org/10.1186/ 1471-2369-14-69.
18 Enomoto A, Kimura H, Chairoungdua A, Shigeta Y, Jutabha P, Cha SH, et al. Molecular identification of a renal urate anion exchanger that regulates blood urate levels. Nature. 2002 May;417(6887):447-52.

19 Liu L, Liu J, Yuan Y, Wang Y, Xu Y, Chen J, et al. The association between elevated serum uric acid levels and islet $\beta$-cell function indexes in newly diagnosed type 2 diabetes mellitus: a cross-sectional study. PeerJ. 2018;6:e4515.

20 Fox IH. Metabolic basis for disorders of purine nucleotide degradation. Metab Clin Exp. 1981 Jun;30(6):616-34.

21 Cook DG, Shaper AG, Thelle DS, Whitehead TP. Serum uric acid, serum glucose and diabetes: relationships in a population study. Postgrad Med J. 1986 Nov;62(733):1001-6. Available from: https://www.ncbi.nlm.nih. gov/pubmed/3628142.http: //dx.doi.org/ 10.1136/pgmj.62.733.1001.

22 Jalal DI, Maahs DM, Hovind P, Nakagawa T. Uric acid as a mediator of diabetic nephropathy. Semin Nephrol. 2011 Sep;31(5):459-65..

23 Mazzali M, Hughes J, Kim YG, Jefferson JA, Kang DH, Gordon KL, et al. Elevated uric acid increases blood pressure in the rat by a novel crystal-independent mechanism. Hypertension. 2001 Nov;38(5):1101-6.

24 Liu P, Chen Y, Wang B, Zhang F, Wang D, Wang Y. Allopurinol treatment improves renal function in patients with type 2 diabetes and asymptomatic hyperuricemia: 3-year randomized parallel-controlled study. Clin Endocrinol. 2015 Oct;83(4):475-82.

25 Mauer M, Doria A. Uric acid and diabetic nephropathy risk. Contrib Nephrol. 2018;192: 103-9. Available from: https://www.karger. com/Article/FullText/484284.

26 Milionis HJ, Kakafika AI, Tsouli SG, Athyros VG, Bairaktari ET, Seferiadis KI, et al. Effects of statin treatment on uric acid homeostasis in patients with primary hyperlipidemia. Am Heart J. 2004 Oct;148(4):635-40. 